\title{
Cinétique de la dégradation dans le rumen des matières azotées et des parois de prairies de Paspalum dilatatum soumises à diverses modalités de pâturage au cours de différentes saisons
}

\author{
Alejandra Acosta*, Graciela Acosta, Juan José Grigera Naón, \\ Víctor Alejandro Deregibus, Alejandra Ayala Torales
}

Facultad de Agronomía, Universidad de Buenos Aires, Av. San Martín 4453,

1417, Buenos Aires, Argentine

(Reçu le ler octobre 1997 ; accepté le 6 juillet 1998)

\begin{abstract}
Kinetics of degradation in the rumen of crude protein and the neutral detergent fibre of Paspalum dilatatum pastures subjected to different grazing frequencies and seasons. Rumen degradability of neutral detergent fibre (NDF) and the crude protein (CP) have been studied in Paspalum dilatatum pastures. The pastures were grazed by six dry Holstein cows during summer and autumn with high frequency $(\mathrm{F})$ : less than $5 \%$ of the total tillers per $\mathrm{m}^{2}$ at blossom, and low frequency (PF): more than $60 \%$ of the total tillers per $\mathrm{m}^{2}$ at blossom. Degradability was measured using the Nylon bag technique. The effective NDF degradability (assuming a rate of passage of $4 \% \cdot \mathrm{h}^{-1}$ ) did not vary significantly between seasons ( 37.4 versus $40.6 \%$ ), being lower in summer. The content of rapidly rumen degradable protein ( 34.9 versus $20.8 \%$ ) and digestion rate of the less degradable CP fraction ( 5.5 versus $4.1 \% \cdot \mathrm{h}^{-1}$ ) were higher in autumn, probably due to the lower NDF content during this season. It was also observed in autumn that the rapidly degradable fraction of the protein was higher with low grazing frequency, due to variation of this fraction in the different parts of the plant. Effective degradability was also higher in the less frequently grazed pasture, which may be associated with a higher rapidly degradable fraction of the protein. In autumn, Paspalum dilatatum pastures with low grazing frequency showed lower values for NDF $(41.3$ versus $57.7 \%)$ and protein degradability $(72.1$ versus $80.7 \%)$ than those pastures without this species. These differences can be explained by morphological characteristics of $\mathrm{C}_{3}$ and $\mathrm{C}_{4}$ species. (@) Elsevier / Inra)
\end{abstract}

grazing / protein / fibre / rumen / degradability

Résumé - Six vaches adultes munies d'une canule du rumen ont été utilisées pour étudier, par la méthode des sachets de Nylon, la cinétique de dégradation des parois et de l'azote de prairies de Paspalum dilatatum soumises, au cours de deux saisons (ćté et automne) à dcux fréquences de pâtu-

* Correspondence and reprints 
rage (pâturage fréquent $(\mathrm{F})$ : moins de $5 \%$ de talles totales de Paspalum par $\mathrm{m}^{2}$ étaient au stade de floraison ; pâturage peu fréquent (PF) : plus de $60 \%$ de talles totales par $\mathrm{m}^{2}$ étaient au stade de floraison). La dégradabilité théorique des parois varie très peu selon les saisons $(37,4 \%$ versus $40,6 \%)$, elle est un peu plus faible en été. Pour l'azote, la fraction rapidement dégradable $(34,9 \%$ versus $20,8 \%$ ) et la vitesse de digestion de la fraction lentement dégradable $\left(5,5 \% \cdot \mathrm{h}^{-1}\right.$ versus $\left.4,1 \% \cdot \mathrm{h}^{-1}\right)$ sont plus élevées en automne, en raison probablement de la teneur en parois plus faible en cette saison. Pendant l'automne, la fraction rapidement dégradable est supérieure avec le pâturage PF. L'explication en serait la variation de la teneur en cette fraction (a) dans les différentes parties de la plante. La dégradabilité théorique est aussi plus élevée dans le cas du pâturage PF et peut résulter de la fraction rapidement dégradable. Pendant l'automne les prairies de Paspalum dilatatum soumises à un pâturage $\mathrm{PF}$ ont des dégradabilités des parois $(41,3 \%$ versus $57,7 \%)$ et de l'azote $(72,1 \%$ versus $80,7 \%)$ plus faibles que celles sans Paspalum dilatatum. Ces différences peuvent s'expliquer par les caractéristiques morphologiques différentes des graminées $\mathrm{C}_{3}$ et $\mathrm{C}_{4}$. (® Elsevier / Inra)

pâturage / azote / paroi / rumen / dégradabilité

\section{INTRODUCTION}

Parmi les graminées $\mathrm{C}_{4}$, Paspalum dilatatum (espèce naturelle de la plaine argentine) se caractérise par sa bonne qualité fourragère et sa tolérance aux gelées [11]. Différentes études indiquent qu'elle a une teneur en matières azotées totales (MAT) acceptable (13-14\%) et des digestibilités d'environ 57 et $61 \%$ pendant l'été et l'automne respectivement $[2,5]$. Cette espèce permet donc d'améliorer l'offre de fourrage en quantité et en qualité pendant l'été et au début de l'automne dans une grande zone, où les limitations édaphiques ne permettent pas de cultiver la luzerne.

Les variations dans la proportion des différents tissus entre graminées tropicales et tempérées sont liées à des mécanismes photosynthétiques différents, $\mathrm{C}_{4}$ et $\mathrm{C}_{3}$ respectivement. Ces différences sont très importantes dans les feuilles, faibles dans les gaines et peu fréquentes dans les tiges [26].

Quand les fourrages vieillissent, leurs teneurs en énergie nette, et leur vitesse de digestion diminuent [24]. De même la teneur en matières azotées diminue et la composition de ces matières azotées est modifiée [14].

Différentes fréquences de défoliation peuvent en outre modifier les caractéristiques morphologiques et chimiques des fourrages pâturés et, par là, la digestion ruminale et la quantité ingérée par l'animal [13].

Pour exploiter correctement un fourrage, il est nécessaire de connaître l'évolution de sa valeur nutritive avec le stade de végétation et comment elle est affectée par différents modes de pâturage.

Pour inclure avec succès le Paspalum dilatatum dans les prairies, il faut évaluer la qualité de ces prairies pour pouvoir les exploiter au mieux. Les hypothèses suivantes ont donc été établies.

- Pendant l'été, le pâturage fréquent de Paspalum dilatatum permet une repousse active du fourrage, qui assure une valeur nutritive élevée ;

- En automne, le développement et la maturité de Paspalum dilatatum dans le pâturage peu fréquent, affecte peu la valeur nutritive du repas, compte tenu que les qualités nutritionnelles des graminées $\mathrm{C}_{4}$ diminuent lentement avec le temps.

L'objectif de cette étude est d'évaluer dans les conditions du pâturage la valeur nutritive de Paspalum dilatatum, en fonction du rythme d'exploitation pendant l'été et l'automne. 


\section{MATÉRIEL ET MÉTHODES}

Le travail s'est déroulé pendant l'été et l'automne 1995 sur différentes prairies au domaine de Castelar (Estación Experimental Agropecuaria : $34^{\circ} 36^{\prime}$ Latitude Sud, $58^{\circ} 40^{\prime}$ Longitude Ouest), où deux essais ont été réalisés. Une prairie composée de Paspalum et fétuque a été divisée en six parcelles de $5000 \mathrm{~m}^{2}$. Dans l'essai I, trois parcelles ont été assignées de façon aléatoire, pour chaque modalité de pâturage. Dans l'essai II, trois parcelles de l'essai I et trois parcelles de la même surface $\left(5000 \mathrm{~m}^{2}\right)$ sans Paspalum dilatatum ont été utilisées.

\section{Essai I :}

L'objectif a été d'étudier la valeur nutritive de l'herbe d'une prairie composée de Paspalum (Paspalum dilatatum, Poir) et de fétuque (Festuca arundinacea, Schreb.) soumise à un pâturage fréquent $(\mathrm{F})$ et peu fréquent $(\mathrm{PF})$ en été et en automne.

Essai II :

Dans ce cas l'objectif a été de comparer la valeur nutritive de l'herbe, pendant l'automne de deux prairies : prairie (A) de Paspalum dilatatum et fétuque et prairie (B) sans Paspalum dilatatum, de fétuque et de brome (Bromus catharticus, Val.) pâturées peu fréquemment $(\mathrm{PF})$. La proportion respective des espèces dans chaque prairie était de $50 \%$.

Les modalités de pâturage, les animaux utilisés et les paramètres evalués ont été les mêmes dans les deux essais.

\subsection{Modalités de pâturage}

Le début du pâturage fréquent $(\mathrm{F})$ a été déterminé quand moins de $5 \%$ de talles totales de Paspalum dilatatum par $\mathrm{m}^{2}$ étaient au stade de floraison.

Le début du pâturage peu fréquent (PF) a été determiné quand plus de $60 \%$ de talles totales de Paspalum dilatatum par $\mathrm{m}^{2}$ étaient au stade de floraison.

Les durées de repos des pâturages (F) et (PF) ont été de 30 et $45 \mathrm{j}$ respectivement.

Le chargement a été determiné afin d'offrir $20 \mathrm{~kg} \mathrm{MS} / \mathrm{animal} / \mathrm{j}$.

\subsection{Animaux}

Six vaches porteuses d'une canule du rumen (poids vif moyen $550 \mathrm{~kg}$ ) ont été utilisées. Les animaux disposaient d'eau à volonté et les essais ont commencé après une période de $10 \mathrm{j}$ d'adaptation au régime.

\subsection{Paramètres évalués}

La méthode des sachets de Nylon proposée par Mehrez et Orskov [15] a été utilisée pour mesurer la cinétique de la digestion. Pour chacune des fréquences de pâturage, au début de chaque essai, un échantillon moyen de $2 \mathrm{~kg}$ de fourrage frais a été prélevé (sur les trois parcelles) à la main en simulant ce que les animaux eux-mêmes pâturaient dans la parcelle. Environ $5 \mathrm{~g}$ de matière sèche (MS) de fourrage frais, coupé en brins de 1 à $1,5 \mathrm{~cm}$ pour simuler la mastication, ont été introduits dans des sachets de Nylon de porosité de $50 \mu \mathrm{m}$, mesurant $7,5 \times$ $20 \mathrm{~cm}$ à l'extérieur et $6 \times 18 \mathrm{~cm}$ à l'intérieur, ce qui conduit à un rapport poids de substrat/surface du sachet de $23 \mathrm{mg} \mathrm{MS} \cdot \mathrm{cm}^{-2}$. Les sachets contenant les échantillons de fourrage frais ont été placés pendant $6,9,12,24,48$ et $72 \mathrm{~h}$ dans le rumen des vaches à raison d'un par vache et par temps d'incubation. Ainsi, pour chaque traitement, chaque point de la cinétique de dégradation est la moyenne de six mesures. À leur sortie du rumen les sachets ont été rincés à l'eau froide jusqu'à obtention d'une eau claire et ont ensuite été séchés à l'étuve à $65^{\circ} \mathrm{C}$ pendant $48 \mathrm{~h}$.

Les résidus obtenus ont été analysés pour déterminer leurs teneurs en NDF (neutral detergent fibre: Goering et Van Soest [10]) et en MAT ( $\mathrm{N}$ total $\times 6,25$, semi-micro Kjeldahl). Les paramètres de la cinétique de digestion du NDF et des MAT ont été calculés à l'aide du programme de Garciarena et Hofer [9], en utilisant pour le NDF l'équation de Mertens et Loften [16] :

$$
y=\text { FPDNDF } * \mathrm{e}^{-(\mathrm{k} d *(t-\mathrm{L}))}+\mathrm{FINDF}
$$

où : $y=$ proportion de NDF restant dans le sachet au temps $t$, FPDNDF $=$ fraction potentiellement digestible $\mathrm{du} \mathrm{NDF}, \mathrm{k} d=$ vitesse de digestion, $\mathrm{L}=$ temps de latence, FINDF $=$ fraction indigestible de NDF, $t=$ temps d'incubation.

La dégradabilité théorique du NDF (DTNDF), a été calculéc en admettant un taux de sortie des particules égal à 0,04 .

$\mathrm{DTNDF}=\mathrm{FPDNDF} * \mathrm{e}^{-\left(k \mathrm{p}^{*} \mathrm{~L}\right)} *(\mathrm{~K} d /(\mathrm{K} d+\mathrm{Kp}))$

Pour les MAT l'équation de Orskov et Mc Donald [20] a été utilisée.

$$
y=a+b^{*}\left(1-\mathrm{e}^{-\left(i^{*} \cdot t\right)}\right)
$$


où :

$y=$ la dégradation de l'aliment incubé pendant le temps $t, a=$ fraction rapidement dégradable, $b=$ fraction de dégradation plus lente, $c=$ vitesse de dégradation de la fraction $\mathrm{b}, t=$ temps d'incubation.

La dégradabilité théorique des MAT (DTMAT) a été calculée en utilisant dans l'équàtion un taux de sortie de $\mathrm{K} p=0,04$.

$$
\text { DTMAT }=a+((b * c) /(c+K p))
$$

\subsection{Analyses statistiques}

Dans l'essai I, les résultats de la composition chimique du fourrage et les paramètres de la cinétique ruminale ont été soumis à une analyse de variance à deux facteurs (saison/fréquence de pâturage) comprenant deux niveaux pour le premier (saison) et deux pour le second (fréquence de pâturage) selon le modèle :

$$
Y_{\mathrm{ijk}}=u+A_{\mathrm{i}}+B_{\mathrm{j}}+I_{\mathrm{ij \textrm {k }}}+e_{\mathrm{ijk}}
$$

où $Y$ est le paramètre étudié ; $u$, la moyenne du paramètre $; A_{\mathrm{i}}$, le facteur saison $(i=1,2) ; B_{\mathrm{j}}$, le facteur fréquence de pâturage $(j=1,2) ; I_{i j \mathrm{k}}$, l'interaction $A^{*} B(k=1,-, 6) ; e_{\mathrm{i} i \mathrm{k}}$, est l'erreur résiduelle du modèle.

Dans l'essai II, les données ont été analysées selon un schéma aléatoire. Le modèle utilisé est :

$$
Y_{\mathrm{ij}}=u+T_{\mathrm{i}}+e_{\mathrm{ij}}
$$

où $Y$ est le paramètre étudié ; $u$, la valeur moyenne du paramètre; $T_{\mathrm{i}}$, l'effet des traitements $(i=1,2) ; e_{\mathrm{ij}}$, est l'erreur résiduelle du modèle.

Pour les deux essais, le logiciel Statistix Version 3.5. [22] a été utilisé. Les moyennes sont comparées par le test de Tukey $(p<0,10)$.

\section{RÉSULTATS}

\section{Essai I}

La composition chimique de la prairie composée de Paspalum et de fétuque proposée aux animaux est donnée dans le tableau I. La saison a affecté la teneur en NDF qui a été plus élevée en été qu'en automne $(80,4 \%$ versus $77,6 \%)$, tandis que la teneur en MAT n'a été supérieure en automne qu'avec le pâturage $F$.

Il existe de même des différences significatives liées à la saison pour les paramètres de dégradation du NDF (tableau $I I$ ). Pendant l'été, les teneurs en fraction potentiellement digestible du NDF (FPDNDF) sont plus faibles et le temps de latence de la dégradation est plus élevé. Il en est de même pour les paramètres de la dégradation ruminale des MAT (tableau III). La vitesse de dégradation (c) de la fraction potentiellement dégradable (b) est plus faible en été qu'en automne quelle que soit la fréquence de pâturage. La fraction rapidement dégradable (a) est plus élevée en automne avec le pâturage PF. La teneur de la fraction potentiellement dégradable est plus élevée en automne qu'en été et il en est de même de la DT $(67,8$ versus $51,7 \%)$.

\section{Essai II}

La composition chimique des fourrages des prairies avec et sans Paspalum, proposés aux animaux à l'automne, est donnée dans le tableau IV. La teneur en NDF a été importante dans la prairie avec Paspalum, alors que celle en MAT a été plus faible.

\begin{tabular}{|c|c|c|c|c|c|c|c|c|}
\hline & \multicolumn{2}{|c|}{ Été } & \multicolumn{3}{|c|}{ Automne } & \multicolumn{3}{|c|}{ Probabilité } \\
\hline & F & PF & $F$ & PF & es & $S$ & $\mathrm{P}$ & $\mathrm{S} \times \mathrm{P}$ \\
\hline $\operatorname{NDF}(\%)$ & 79,8 & 81,0 & 76,8 & 78,5 & 1,2 & 0,04 & 0,16 & 0,77 \\
\hline $\operatorname{MAT}(\%)$ & 11,0 & 11,0 & 13,0 & 11,0 & 0,2 & 0,04 & 0,04 & 0,04 \\
\hline
\end{tabular}

Tableau I. Teneur en NDF et MAT d'une prairie de Paspalum et fétuque avec deux fréquences de pâturage (fréquent, $\mathrm{F}$ et peu lréquent, $\mathrm{PF}$ ) en été et en automne.

NDF : neutral detergent fibre, MAT : matières azotées totales, es : erreur standard, $\mathrm{S}:$ Saison, $\mathrm{P}:$ Pâturage, $\mathbf{S} \times \mathbf{P}$ : interaction, niveau de signification $p<0,10$. 
Tableau II. Paramètres de la digestion ruminale du NDF d'une prairie de Paspalum et fétuque avec un pâturage fréquent $(\mathrm{F})$ et peu fréquent $(\mathrm{PF})$ en été, et en automne.

\begin{tabular}{|c|c|c|c|c|c|c|c|c|}
\hline & \multicolumn{2}{|c|}{ Été } & \multicolumn{3}{|c|}{ Automne } & \multicolumn{3}{|c|}{ Probabilité } \\
\hline & $\mathrm{F}$ & PF & $\mathrm{F}$ & $\mathrm{PF}$ & es & $\mathrm{S}$ & $P$ & $\mathbf{S} \times \mathbf{P}$ \\
\hline $\mathrm{c}\left(\% \cdot \mathrm{h}^{-1}\right)$ & 5,0 & 4,4 & 4,6 & 4,4 & 0,3 & 0,66 & 0,54 & 0,68 \\
\hline FPDNDF (\%) & 75,7 & 73,0 & 79,5 & 79,2 & 1,1 & 0,01 & 0,53 & 0,60 \\
\hline $\mathrm{T}$ de lat. (heure) & 1,5 & 0,9 & 0,1 & 0 & 0,4 & 0,06 & 0,65 & 0,74 \\
\hline DT $(\%) \#$ & 39,0 & 35,9 & 42,0 & 41,3 & 0,8 & 0,38 & 0,36 & 0,55 \\
\hline
\end{tabular}

c : vitesse de dégradation, FPDNDF : fraction potentiellement digestible, $\mathrm{T}$ de lat. : Temps de latence, DT : dégradabilité théorique, \# : taux de passage $4 \% \cdot \mathrm{h}^{-1}$, es : erreur standard, $\mathrm{S}:$ Saison, $\mathrm{P}: \mathrm{Pâturage}, \mathrm{S} \times \mathrm{P}:$ interaction, niveau de signification $p<0,10$.

Tableau III. Paramètres de la digestion de MAT d'une prairie de Paspalum et fétuque au pâturage fréquent (F) et peu fréquent (PF) en été et en automne.

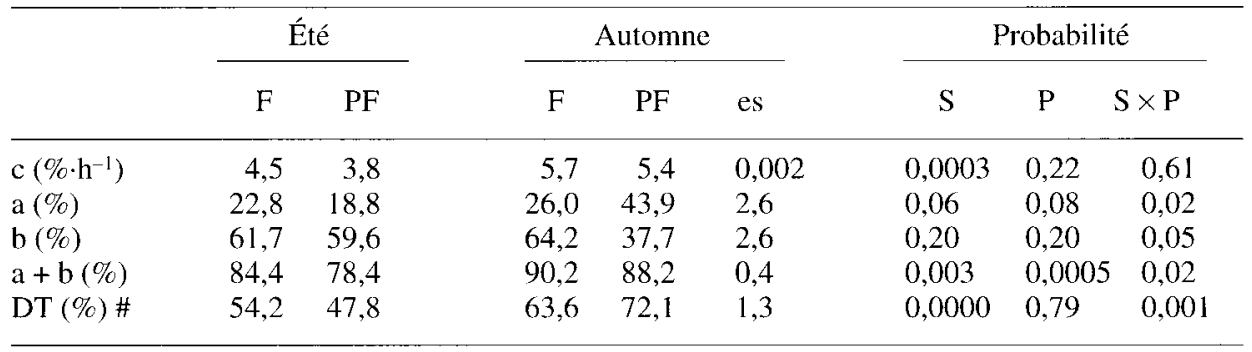

$\mathrm{c}:$ vitesse de dégradation, $\mathrm{a}:$ fraction rapidement dégradable, $\mathrm{b}:$ fraction lentement dégradable, $\mathrm{a}+\mathrm{b}:$ fraction potentiellement dégradable, DT : dégradabilité théorique, \# : taux de passage : $4 \% \cdot \mathrm{h}^{-1}$, es : erreur standard, $\mathrm{S}$ : Saison, $\mathrm{P}$ : Pâturage, $\mathrm{S} \times \mathrm{P}$ : interaction, niveau de signification $p<0,10$.

Les paramètres de la digestion ruminale du NDF, c, FPDNDF et DT (tableau $V$ ) sont tous plus élevés avec la prairie sans Paspalum dilatatum .

En revanche, pour la dégradation ruminale de l'azote (tableau VI), seuls les paramètres $c, a+b$, et DT sont plus élevés pour la prairie sans Paspalum alors que les paramètres $a$ et $b$ ne sont pas significativement différents.

\section{DISCUSSION}

\section{Essai I}

La teneur plus faible en FPDNDF en été est vraisemblablement due aux témperatures
Tableau IV. Teneurs en NDF et en MAT des prairies avec et sans Paspalum pendant l'automne.

Prairies

Avec Paspalum Sans Paspalum

\begin{tabular}{lll}
\hline $\operatorname{NDF}(\%)$ & $79,5^{\mathrm{a}}$ & $73,0^{\mathrm{b}}$ \\
$\operatorname{MAT}(\%)$ & $11,0^{\mathrm{a}}$ & $16,0^{\mathrm{b}}$ \\
\hline
\end{tabular}

NDF : neutral detergent fibre, MAT : matières azotées totales ; ${ }^{a}, b$ traitements significativement différents $(p<0,10)$.

plus élevées qui augmentent non seulement les teneurs en NDF mais aussi la lignification des parois [27]. 
Tableau V. Paramètres de la digestion ruminale de la paroi cellulaire (NDF) des prairies avec et sans Paspalum pendant l'automne.

\begin{tabular}{lcccc}
\hline & \multicolumn{2}{c}{ Prairies } & & \\
\cline { 2 - 3 } & Avec Paspalum & Sans Paspalum & es & Probabilité \\
\hline $\mathrm{c}\left(\% \cdot \mathrm{h}^{-1}\right)$ & 4,4 & 6,5 & 0,6 & 0,029 \\
FPDNDF $(\%)$ & 79,2 & 89,5 & 1,6 & 0,0013 \\
DT $(\%) \#$ & 41,3 & 57,7 & 2,2 & 0,001 \\
\hline
\end{tabular}

$\mathrm{c}:$ vitesse de dégradation, FPDNDF : fraction potentiellement digestible, DT : dégradabilité théorique, \# : taux de passage $4 \% \cdot h^{-1}$, es : erreur standard, niveau de signification $p<0,10$.

Tableau VI. Paramètres de la digestion ruminale de l'azote (MAT) des prairies avec et sans Paspalum pendant l'automne.

\begin{tabular}{lcccc} 
& \multicolumn{3}{c}{ Pâtures } & \\
\cline { 2 - 3 } & Avec Paspalum & Sans Paspalum & es & Probabilité \\
\hline $\mathrm{c}\left(\% \cdot \mathrm{h}^{-1}\right)$ & 5,4 & 7,7 & 0,6 & 0,025 \\
$\mathrm{a}(\%)$ & 50,5 & 53,4 & 5,2 & 0,70 \\
$\mathrm{~b}(\%)$ & 37,7 & 41,0 & 5,4 & 0,677 \\
$\mathrm{a}+\mathrm{b}(\%)$ & 88,2 & 94,4 & 0,7 & 0,0002 \\
DT $(\%) \#$ & 72,1 & 80,7 & 1,4 & 0,0019 \\
\hline
\end{tabular}

$\mathrm{c}:$ vitesse de dégradation, $\mathrm{a}:$ fraction rapidement dégradable, $\mathrm{b}:$ fraction lentement dégradable, $\mathrm{a}+\mathrm{b}:$ fraction potentiellement dégradable, DT : dégradabilité théorique, es : erreur standard, \# : taux de passage : $4 \% \cdot \mathrm{h}^{-1}$, niveau de signification $p<0,10$.

La teneur plus élevée du NDF des fourrages en été doit aussi expliquer la diminution de la fraction (a) des MAT, l'augmentation de la fraction (b) qui est constituée essentiellement par la fraction des MAT liées au NDF (N-NDF) et la diminution de la vitesse de dégradation (c) de cette fraction. Funk et al, [8] ont montré que la fraction (a) diminue lorsque la maturité du fourrage augmente ; Aii et Stobbs [3] ont observé cette même tendance mais sans différence significative. Les données de notre essai sont en accord avec ces observations pour les fourrages d'été mais pas d'automne. La raison en serait la variation de la teneur de la fraction (a) de l'azote dans les différentes parties de la plante. Cette fraction (a) est plus élevée dans les tiges et les inflores- cences que dans les feuilles. Dans notre essai le chargement a été conduit pour éviter un tri important et les animaux ont dû ainsi consommer des tiges et des inflorescences.

Les résultats obtenus pour la fraction potentiellement dégradable corroborent ceux de Napoli [18], Vogel et al., [25] et Elizalde [6], qui ont observé une diminution de cette fraction avec l'avancement de la maturité de la plante.

Les valeurs de dégradabilité théorique sont en accord avec celles obtenues en Argentine [19, 21]. Dans cet essai, les valeurs inférieures en été sont liées à la diminution de la qualité fourragère de la plante avec l'avancement de la maturité, à un moindre contenu en MAT et à une valeur supérieure en NDF (tableau I). 


\section{Essai II}

Les valeurs de la digestion ruminale du NDF sont plus élevées avec la prairie sans Paspalum. Ceci peut être attribué d'une part à la teneur plus faible en NDF de la prairie sans Paspalum $(73,0 \%$ versus $79,5 \%)$, d'autre part aux caractéristiques morphologiques différentes des graminées $C_{3}$ et $C_{4}$. La digestibilité plus élevée des graminées $\mathrm{C}_{3}$ est produite par des différences de structure anatomique liées à des mécanismes photosynthétiques différents [17]. Différents auteurs [4, 23] ont montré que le mésophylle des graminées $\mathrm{C}_{4}$ est digéré plus lentement que celui des graminées $C_{3}$. Les graminées $\mathrm{C}_{4}$ ont aussi une proportion plus élevée d'épiderme et une quantité supérieure de vaisseaux, tissus dont la digestion plus lente et partielle constituent une barrière physique à la dégradation microbienne. En outre les graminées $\mathrm{C}_{4}$ ont une teneur en lignine plus élevée que les graminées $\mathrm{C}_{3}$ [7].

La vitesse de digestion de l'azote plus élevée dans la prairie sans Paspalum, résulterait de la vitesse de digestion du NDF plus élevée $\left(4,44\right.$ versus $\left.6,50 \% \cdot \mathrm{h}^{-1}\right)$, cette relation est en accord avec celle mesurée par Napoli et Santini [19]. La vitesse de digestion et le pourcentage de la fraction potentiellement dégradable de l'azote plus élevés dans la prairie sans Paspalum expliquent que la dégradabilité théorique des MAT soit, elle aussi, plus élevée. Des résultats semblables ont été obtenus par Acosta et al. [1] en utilisant des moutons maintenus en cases individuelles et recevant du fourrage de mêmes caractéristiques que celui employé dans cette étude. Finalement, il est intéressant de remarquer que malgré des teneurs en MAT très différentes, les fourrages des deux prairies fournissent à l'animal la même quantité de MAT échappant à la dégradation dans le rumen $110(1-0,721)=30,7 \mathrm{~g}$, $160(1-0,807)=30,9 \mathrm{~g} \cdot \mathrm{kg}^{-1} \cdot \mathrm{h}^{-1}$ de MS ingerée (tableau VI). Si on admet, en première approche [12] que la fraction $100-(a+b)$ peut être considérée comme un index de la fraction non digestible des MAT du fourrage échappant à la dégradation ruminale, la fourniture de protéines d'origine alimentaire à l'animal serait $30,7-11,8=18,9 \mathrm{~g} \cdot \mathrm{kg}^{-1}$ de MS pour la prairie avec Paspalum et $30,9-5,60=25,30 \mathrm{~g} \cdot \mathrm{kg}^{-1}$ de MS pour la prairie sans Paspalum. Ces résultats demandent cependant à être confirmés.

\section{CONCLUSIONS}

L'effet de la saison est plus marqué que la modalité de pâturage pour les paramètres de la cinétique de dégradation ruminale dans les prairies composées de graminées $\mathrm{C}_{3}$ et $\mathrm{C}_{4}$. L'inclusion de Paspalum dilatatum dans les prairies affecte peu la valeur nutritive de l'herbe ingérée à l'automne.

\section{RÉFÉRENCES}

[1] Acosta A., Acosta G., Grigera Naon J., Deregibus V., Ayala Torales A., Soria F., Evaluación de la calidad nutricional de pasturas con inclusión de pasto miel a través de los paráme. tros del ambiente ruminal, Rev. Arg. Prod. Anim. 15 (1995) 477-479.

[2] Acosta G., Deregibus V., Hammar R., Inclusión de Pasto Miel (Paspalum dilatatum, Poir) en pasturas. 2. Efecto sobre el valor nutritivo, Rev. Arg. Prod. Anim. 16 (1996) 157-167.

13] Aii T., Stobbs T., Solubility of the protein of tropical pasture species and the rate of its digestion in the rumen, Anim. Feed Sci. Tech. 5 (1980) 183-192.

14] Akin D., Histological and physical factors affecting digestibility of forage. Agronomy J, 81 (1989) 17-25.

[5] Cicardini E., Irazoqui J., Orbea J., Curvas de producción y calidad del forraje de ocho ecotipos de Pasto Miel (Paspalum dilatatum, Poir), Rev. Arg. Prod. Anim. 4 (1984) 411-421.

[6] Elizalde J.C., Suplementación con silo de maiz en vacunos en pastoreo de avena, ambiente ruminal y dinamica de digestión, tesis M.S., Universidad Nacional de Mar del Plata Facultad de Ciencias Agrarias Balcarce, Argentina, (1990) pp. 110

[7] Ford C., Morrison I., Wilson J., Temperature effects on lignina, hemicellulose and cellulose in tropical and temperate grasses, Australian J. Agr. Res. 30 (1979) 621-633.

[8] Funk M., Galyean M., Branine M., Krysl L., Steers grazing blue grama rangeland throughout the growing season. I. Dietary composition, intake, digesta kinetics and ruminal fermentation, J. Anim. Sci. 65 (1987) 1342-1353. 
[9] Garciarena D., Hofer C., Programa para el cálculo de modelos de dinámica de la digestión ruminal, EEA-INTA, Concepción del Uruguay, Entre Rios, Argentina, 1993, 12 p.

[10] Goering H., Van Soest P., Forage fiber analysis (apparatus, reagents, procedures and some applications), Agric. Handb. 379, ARS, USDA, Washington DC, 1970, 1-20.

[11] Hutton E., Plant breeding and genetics, in: Staf CSIRO (éd.), Some concepts and methods in sub-tropical pasture research, Brisbane, Australia, 1968.

[12] Le Goffe P., Vérite R., Peyraud J.-L., Influence de l'espèce et la saison sur la dégradabilité de l'azote des fourrages verts dans le rumen, Ann. Zootech. 42 (1993) 3-15.

[13] Lemaire G., Chapman D., Tissue Flows in Grazed Plant Communities, in: Hodgson H., Illius A. (éd.) The Ecology and Management of Grazing Systems CAB International UK., 1996, pp. 3-36.

[14] Lyttleton J., Proteins and Nucleic Acids, in: Chemistry and Biochemistry of Herbage, Academic Press, New York, 1973, 63-106.

[15] Mehrez A., Orskov E., A study of the artificial bag technique for determining the digestibility of the feeds in the rumen, Agric Sci Camb 88 (1977) 645-650.

[16] Mertens D., Loften J., The effect of starch on forage fibre digestion kinetics in vitro, J. Dairy Sci. 63 (1980) 1437-1446.

[17] Minson D., Digestible Energy of Forage, in: Forage in Ruminant Nutrition, Academic Press, New York, 1990, 85-149.

[18] Napoli G., Efecto de la suplementación energético-proteica sobre la digestión ruminal de forrajes frescos en condiciones de pastoreo.Tesis M.S. Universidad Nacional de Mar del Plata Facultad de Ciencias Agrarias Balcarce, Argentina, 1987, 114 p.

[19] Napoli G., Santini F., Estimación de la proteína metabolizable en forrajes frescos, Rev. Arg. Prod Anim. 8 (1988) 15-19.

[20] Orskov E., Mac Donald I., The estimation of protein degradability in the rumen from incubation measurement weighted according to rate of passage, J. Agric. Sci. Camb. 92 (1979) 499-503.

[21] Pasinato A., Rearte D., Santini F., Contenido de materia seca del forraje . 1. Ambiente ruminal y dinámica de lá digestión, Rev. Arg. Prod. Anim. 13 (1993) 1-8.

[22] Statistix Version 3.5 (1991) Analytical Software.

[23] Susmel P., Filacorda S., Biological methods to predict the nutritive value of tropical feedstuffs: a critical review, Ann. Zootech 45 (1996) Suppl., $39-51$.

[24] Van Soest P., Environment and forage, in: Van Soest P. (ed.), Nutritional Ecology of the Ruminant, ISBNC-9601586-O-X, Library of Congress Catalog. Card. No 81-83655, O \& B Books, Inc. 1215 NW Kline Place, Corvallis, Oregon 97330 USA, 1982, $374 \mathrm{p}$.

[25] Vogel G.J., Phillips W.A., Horn G.W., Zorrilla Rios $\mathbf{J}$., Effects of forage maturity and microbial contamination of in situ forages residues on kinetics of wheat disappearance, ADSA/SAS An. Meet. Abst., 692 (1989) 284.

[26] Wilson J.R., Cell wall characteristics in relation to forage digestion by ruminants, J. Agric. Sci. 122 (1994) 173-182.

[27] Wilson J.R., Minson D.J., Prospects for improving the digestibility and intake of tropical grasses, Tropical Grass 14 (1980) 253-259. 